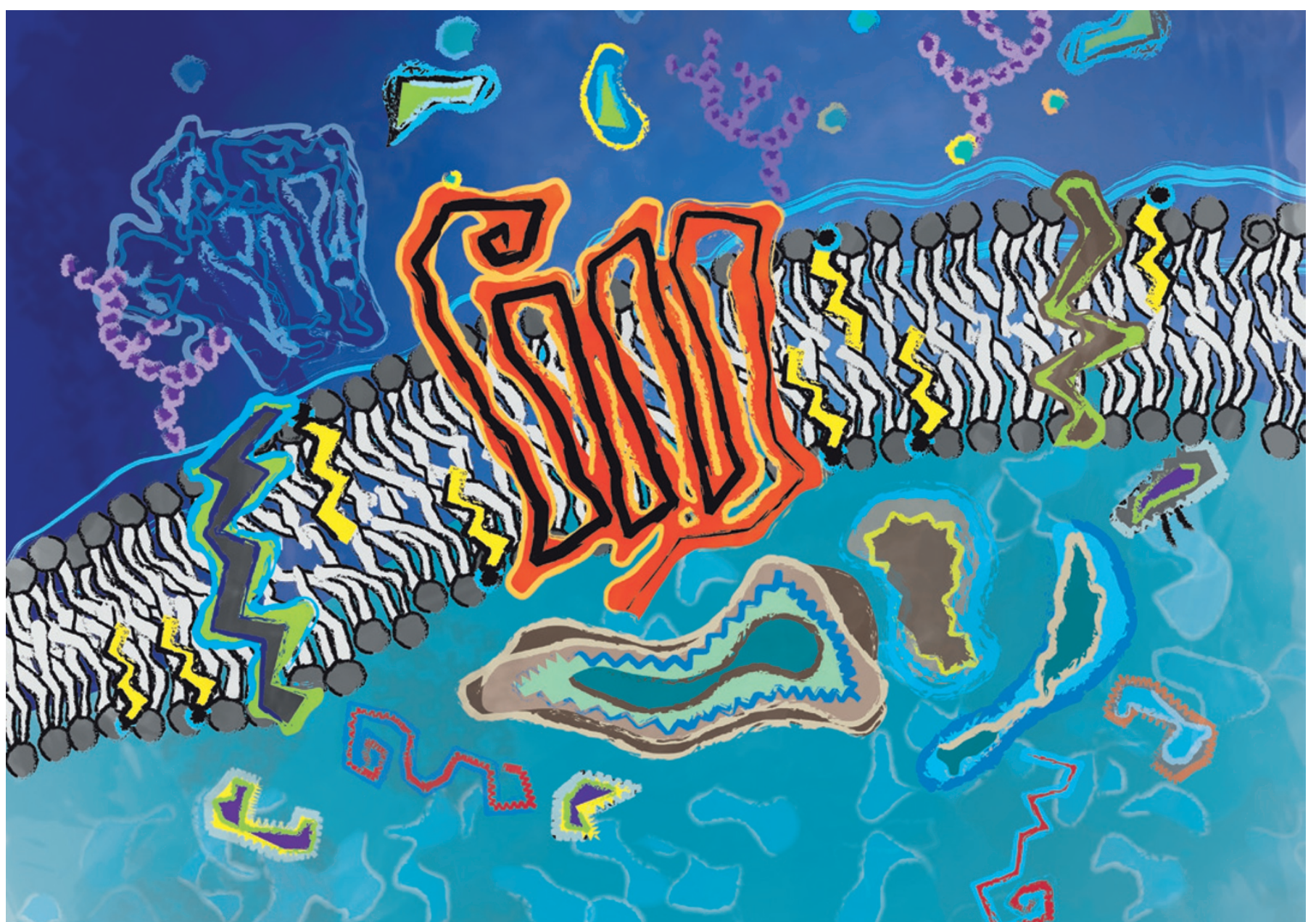

An G-protein-coupled receptor with its seven transmembrane domains, by Katya Kadyshevskaya of the Scripps GPCR Network

\title{
DRUG DISCOVERY
}

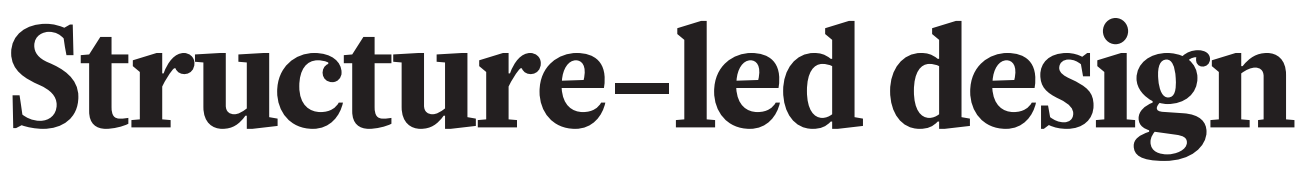

Knowing the structures of G-protein-coupled receptors, says Lindau keynote speaker Brian Kobilka, should help with drug development. But how is this progressing?

\section{BY MONICA HOYOS FLIGHT}

$\mathrm{B}$ rian Kobilka, with his medical background, said he felt like the "the new kid in the chemistry club" at this summer's Lindau Meeting. But it's likely that every attendee was already familiar with his Nobel prizewinning work on the structure of the G-protein-coupled receptor (GPCR) for the hormone adrenaline. This research has "revolutionized the hunt for new GPCR-ergic drugs", and has the potential to improve the treatment of many diseases, says Bryan Roth, a structural biologist and molecular pharmacologist at the University of North Carolina in Chapel Hill.

GPCRs regulate the response of virtually every cell in the body to a wide variety of signals, including hormones, neurotransmitters, ions, odour molecules and even light. When they are activated by the right molecule (or ligand) GPCRs undergo a structural change that triggers a chain of reactions in the cell. By targeting different members of the GPCR family, drugs can have a huge variety of physiological effects. For example, beta blockers can slow the heart rate; antihistamines can prevent allergic reactions; and antipsychotics can modulate neurotransmission. As such, GPCRs present a wealth of opportunities for drug development. Indeed, it is their importance as targets of intensive-care medicines that lured Kobilka out of the clinic and into the lab.

Lefkowitz and Kobilka's work revealed the common structure that underlies the GPCR family as well as the variation among its members (see 'Eureka moment'). Armed with this knowledge, medicinal chemists are starting to design novel ligands that bind to a specific receptor and alter its activity more effectively than existing drugs. This work transcends disciplinary boundaries. According to Arthur Christopoulos, a pharmacologist at Monash University, Melbourne, Australia, translating the biological data into advances in drug discovery will require "biologists and chemists to work closely and speak a common language". Christopoulos is one of many researchers who are aiming to use the emerging information about GPCRs to end the reign of "blunt hammer" $\rightarrow$ NATURE.COM

For some of the latest research on GPCRs, see:

go.nature.com/7rpf8y 


\section{EUREKA MOMENT \\ The small discovery that hinted at something bigger}

In 1968, Robert Lefkowitz tagged hormones with radioactive iodine molecules and showed that the hormones could bind to a receptor outside a cell and trigger a reaction inside it - demonstrating for the first time the existence of biologically active receptors. Brian Kobilka joined Lefkowitz's team in the 1980s with the aim of isolating the gene that encodes the receptor for the hormone adrenaline, which has a key role in the regulation of cardiovascular function. In 1986, they succeeded in cloning the $\beta 2$-adrenergic receptor and realized that it was very similar to the light-detecting receptor, rhodopsin; both had multiple membrane-spanning domains and large hydrophobic stretches. This similarity suggested that the $\beta$-adrenergic receptor was part of a bigger family of receptors that are structurally related but have completely

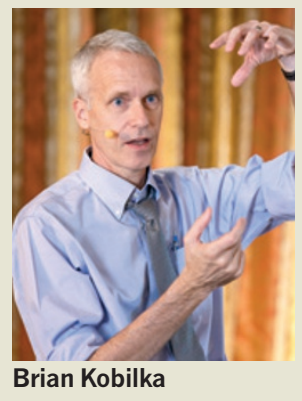

different functions. These soon became known as G-protein-coupled receptors (GPCRs). Since this 'eureka' moment, more than 800 genes encoding GPCRs have been identified, and 23 structures have

been solved (see 'Time and Space). Most of these structures are for receptors in the class A (Rhodopsin-like) family, which includes adrenergic, muscarinic and opioid receptors — established targets for drugs that treat cardiac or respiratory arrest and pain. But GPCRs are notoriously difficult to isolate and crystallize. Not only are they highly insoluble but they are also dynamic — their structure is constantly changing as they interact with different ligands and intracellular signalling proteins. It was only in 2011 that Kobilka managed to crystalize the human $\beta 2$-adrenergic receptor at the exact moment of activation when, bound to a ligand and a G protein, it sends a signal into the cell ${ }^{4}$.

Recently, the structures of receptors from different families have been reported. These include the smoothened receptor (SMO), the first in the frizzled class ${ }^{5}$; and two from class $B$ - the corticotropin-releasing factor 1 receptor (CRF1R) and the glucagon receptor $^{6,7}$. These receptors are clinically validated targets for diseases such as cancer and type 2 diabetes but, owing to their large ligand-binding surfaces, conventional approaches have struggled to generate small-molecule drugs against them. M.H.-F. medicines; they hope to design new drugs targeting GPCRs that will be potent enough to offer better treatment and selective enough to eliminate side-effects.

\section{A PRECISION TOOL}

Drug development is slow and expensive. Fewer than $5 \%$ of the molecules that enter phase I clinical trials are eventually approved by the US Food and Drug Administration; each drug that makes it is estimated to cost its manufacturer close to US\$1 billion. Until recently most drugs, including those that target GPCRs, were formulated with only a limited understanding of the underlying structure and biology of their targets.

The most successful attempts at GPCR drug discovery have targeted receptors that are activated by small molecules such as adrenaline or serotonin, for which highthroughput screening of chemical entities has found analogues. The adrenaline receptor agonist salbutamol, for example, was first approved 45 years ago and remains one of the most effective agents for asthma. However, it is more challenging to develop small molecules with drug-like properties that fit the binding sites of GPCRs with larger protein or peptide ligands. And, given the underlying similarity among GPCRs, it is nearly impossible to identify selective drugs that don't have any unanticipated off-target actions.

Researchers hope that working backwards from a receptor's structure will advance the development of new drugs. Several companies are using structural information about receptors' binding pockets to feed into virtual screening programs, sometimes building drugs

up from smaller fragments. By identifying regions of the binding pocket that are unique to each receptor, researchers can design highly selective ligands - the first step in the development of drugs for targets that were previously considered undruggable. "These approaches can lead to highly optimized drug candidates with improved efficiency," says Fiona Marshall, chief scientific officer of drug discovery company Heptares Therapeutics, based in Welwyn Garden City, UK.

GPCRs, says Marshall, are also emerging as targets for rare diseases that currently lack any drugs at all. So-called 'orphan receptors' (GPCRs for which the natural ligand is not known) are also attractive drug targets. Heptares is working on the structure of recently 'de-orphanized' receptors and has identified agonists for GPR39, which is involved in islet cell function and is a target for diabetes therapies. "This receptor is particularly interesting in that it has the potential to halt the

"These approaches can lead to highly optimized drug candidates with improved efficiency." progression of the disease," she explains. In addition, Heptares is using stabilized pure GPCR preparations to generate antibodies that either activate or silence receptors with large binding pockets, in collaboration with companies such as MorphoSys and MedImmune. These therapeutic antibodies offer many advantages as drugs. "Antibodies can have unique specificity and a long-lasting action, and they can be coupled to other modalities such as drug conjugates for cancer," says Marshall. A number of such antibodies developed by other companies are in clinical trials ${ }^{1}$ and mogamulizumab, a drug marketed as Poteligeo by Tokyo-based biotech company Kyowa Hakko Kirin, has already been approved in Japan for the treatment of adult T-cell leukaemia.

\section{CHANGING LANDSCAPE}

Structural research has revealed other layers of complexity for GPCRs. In addition to the binding site for the endogenous ligand (orthosteric site), GPCRs have allosteric sites, where other molecules can bind. Allosteric sites are more structurally diverse than orthosteric sites, and provide opportunities to fine-tune the activity of GPCRs. However, it is hard to quantify allosteric effects, especially when, for many GPCR targets, the level of signalling needed for optimal therapeutic benefit is unknown. This is an exciting area: "If we can understand how receptor signalling links to clinical outcomes, we will have a better handle on the desired signalling properties of drugs," explains Patrick Sexton, a pharmacologist at Monash University in Melbourne, Australia.

Two allosteric ligands are currently approved as therapeutic agents: cinacalcet (marketed as Sensipar by Amgen, based in Thousand Oaks, California), which activates calcium-sensing receptors and is used to treat hyperparathyroidism; and maraviroc (marketed as Celsentri/Selzentry by UK-based ViiV Healthcare), which blocks the chemokine receptor used by HIV to gain entry into host cells. A number of small companies are investigating allosteric modulators of GPCRs for the treatment of neurodegenerative and 


\section{TIME AND SPACE}

The number of solved structures is rapidly increasing, mainly owing to the efforts of the Scripps GPCR Network. Established in 2008, the network is a large-scale collaboration involving more than a dozen academic and industrial labs. Its goal is to characterize at least 15 GPCRs by 2015 ; it has already solved 13.

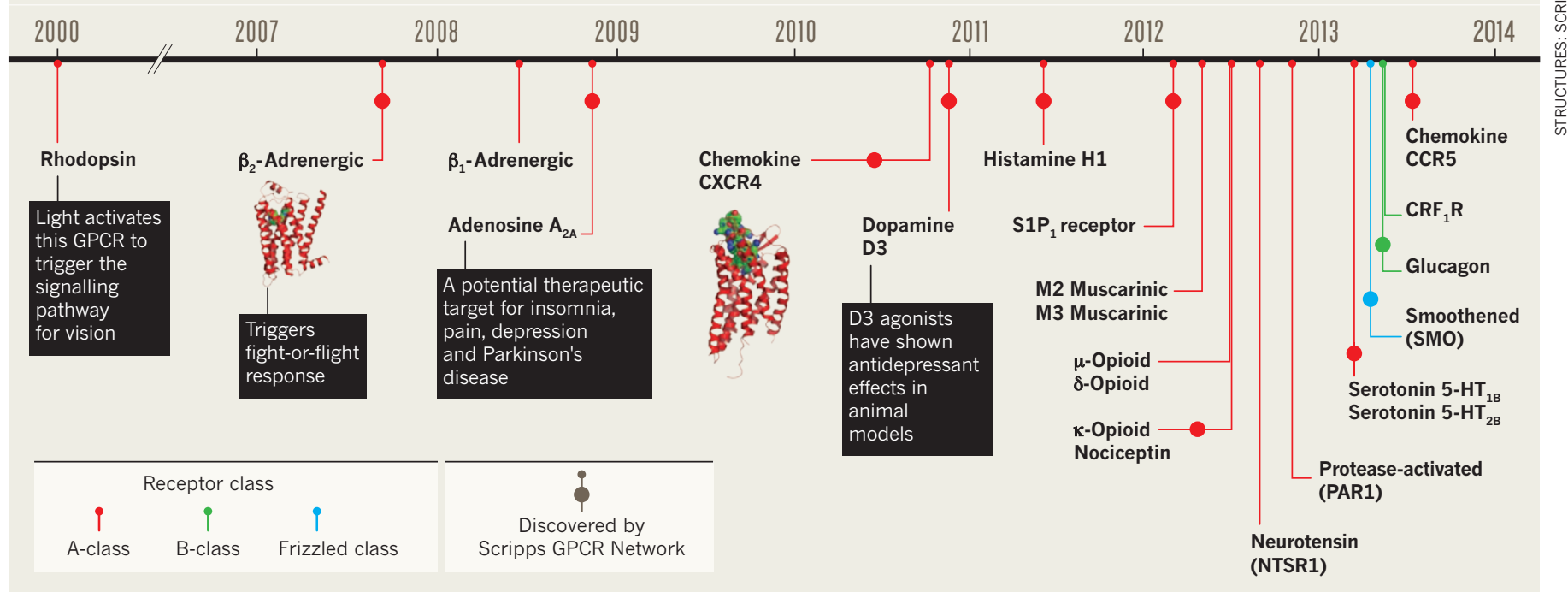

psychiatric diseases. Addex Therapeutics, based in Geneva, has two such compounds in phase II trials, targeted at different metabotropic glutamate receptors (excitatory receptors on neurons, also called mGluRs). Dipraglurant is an inhibitor of mGluR5 that can reverse levodopa-induced dyskinesia in patients with Parkinson's disease and is being evaluated as a treatment for rare dystonias; and ADX71149, which activates mGluR2, can reduce anxiety and the negative symptoms of schizophrenia and is being developed with partner Janssen Pharmaceuticals. "These compounds show great receptor subtype selectivity compared to orthosteric ligands," says Tim Dyer, Chief Executive Officer of Addex.

Further drug selectivity might be achieved with compounds that act simultaneously on both orthosteric and allosteric binding sites. Although such bitopic ligands have been identified in the lab, none has entered clinical trials. Christopoulous has suggested that this strategy might work against the M1 muscarinic acetylcholine receptor, a promising target for the treatment of cognitive deficits that has so far proved undruggable ${ }^{2}$. However, research on bitopic ligands is still in the early stages. Among the most important challenges, says Christopoulous, is figuring out how best to link the two ligands for the orthosteric and allosteric sites. Such conjoined molecules might be "too large to be appropriately optimized and developed as potential drug leads", he adds.

\section{SIGNAL SNAPSHOTS}

Structural studies have shown that GPCRs can adopt different conformations depending on which ligand they are bound to. What's more, the structure adopted by the receptor influences the signalling pathway that

is activated in the cell. The main pathways go through guanosine nucleotide-binding proteins ( $G$ proteins) to trigger metabolic processes. But GPCRs can also bind to arrestin and prevent the activation of $G$ proteins, causing the receptor to retreat inside the cell or to activate alternative signalling pathways. Ligands that trigger just one of these pathways are said to induce biased signalling.

Sexton is investigating whether biased signalling can lead to better drugs. For example, he suspects that the reason why the beta-blocker carvedilol is more effective than other drugs in the same class is because it can induce signalling through arrestins. How this leads to a greater therapeutic benefit is unclear but, "as we understand more about the link between signalling

\section{"The next five} years will see an increase in the number of receptors crystallized." and physiology we can expect increasing numbers of programmes to develop biased ligands", he says. To this end, his lab is examining the effects of such ligands in different tissues and in transgenic animals with receptors that have been modified to respond to natural ligands with biased signalling.

Roth's lab is focusing on the design of GPCR drugs that activate arrestin. "Already we have evidence that such drugs have the potential to revolutionize treatment for a number of common diseases including schizophrenia, cardiovascular disease and chronic pain," he says. Roth is working with Jian Jin, a medicinal chemist at the University of North Carolina who is in the interdisciplinary center for Integrative Chemical Biology and Drug Discovery. Roth and Jin recently described the first $\beta$-arrestin-biased dopamine D2 receptor agonists, which have robust antipsychotic druglike activity in preclinical models ${ }^{3}$. "We are in the process of evaluating potential clinical candidates," Roth says. "Things are progressing nicely."

Nicely - but slowly: drug discovery and development based on GPCR structures "are at an early stage", says Marshall. There are numerous targets and they are difficult to crystallize. What's more, these GPCR structures are static snapshots of receptors that are in constant flux. "The dynamic nature of GPCRs should be factored into computational approaches towards drug design," notes Christopoulos. "Otherwise we will run into the same problems with low success rates that others have had in the past."

Still, Sexton is optimistic and predicts that the next five years will see an increase in both the number of receptors crystallized and the quantitative tools used to describe effects such as signal bias and allostery. These, he adds, "will progressively have an impact on drug candidate selection". As the crossover between biology and chemistry continues, it is unlikely that Kobilka will remain the new kid in the chemistry club for long.

Monica Hoyos Flight is a writer and editor on Nature Reviews Neuroscience and Nature Reviews Drug Discovery in London.

1. Hutchings, C. J., Koglin, M. \& Marshall, F. H. MAbs 2, 594-606 (2010)

2. Davie, B. J., Christopoulos, A. \& Scammells, P.J. ACS Chem. Neurosci. 4, 1026-1048 (2013)

3. Chen, X. et al. J. Med. Chem. 55, $7141-7153$ (2012).

4. Rasmussen, S. G. F. et al. Nature 477, 549-555 (2011)

5. Wang, C. et al. Nature 497, 338-343 (2013).

6. Hollenstein, K. et al. Nature 499, 438-443 (2013).

7. Siu, F. Y. et al. Nature 499, 444-449 (2013). 